\title{
Multiple Lesions With Recurrent Bleeding
}

\author{
Diem-Phuong D. Dao, BS; Kimberly Salkey, MD
}



A 63-year-old man with frontal bossing and a history of jaw cysts presented with numerous lesions on the scalp, trunk, and legs with recurrent bleeding. Both of his siblings had similar findings. Many lesions had been present for at least 40 years. Physical examination revealed a large, irregular, atrophic, hyperpigmented plaque on the central scalp with multiple translucent hyperpigmented papules at the periphery (top). Similar papules and plaques were present at the temples, around the waist, and on the distal lower extremities, lead-

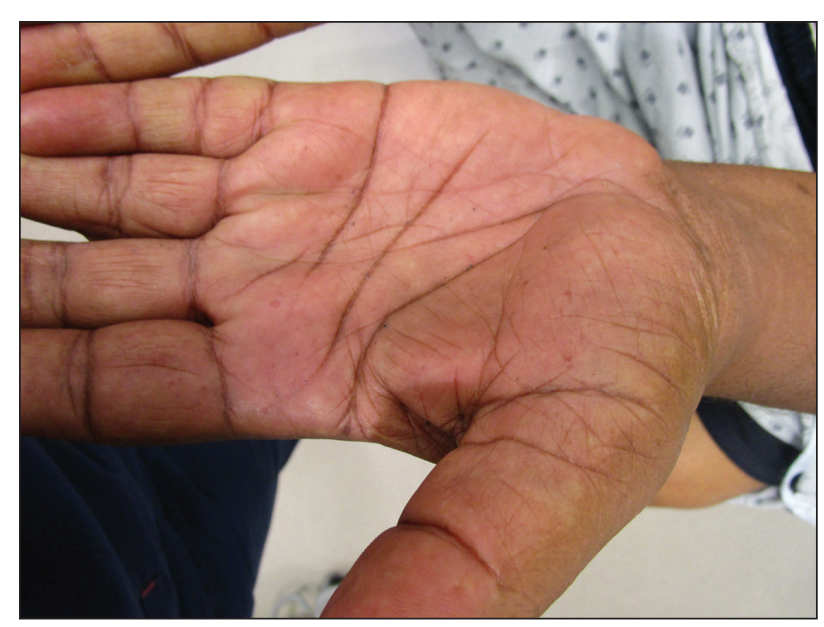
ing to surgical excision of the largest leg lesions. In addition, there were many pinpoint pits on both palms (bottom). A biopsy was submitted for review, which confirmed basal cell carcinomas on the scalp.

\section{WHAT'S YOUR DIAGNOSIS?}
a. Bazex syndrome
b. Muir-Torre syndrome
c. nevoid basal cell carcinoma syndrome
d. psoriasis
e. Rombo syndrome

PLEASE TURN TO PAGE E28 FOR THE DIAGNOSIS

From Virginia Commonwealth University, Richmond. Ms. Dao is from the School of Medicine, and Dr. Salkey is from the Department of Dermatology. The authors report no conflict of interest.

Correspondence: Kimberly Salkey, MD, 401 N 11th St, Ste 520, Box 980164, Richmond, VA 23298-0164 (kimberly.salkey@vcuhealth.org). doi:10.12788/cutis.0409 


\section{THE DIAGNOSIS:}

\section{Nevoid Basal Cell Carcinoma Syndrome}

N evoid basal cell carcinoma syndrome (NBCCS), also known as Gorlin syndrome, is a rare autosomal-dominant disorder that increases the risk for developing various carcinomas and affects multiple organ systems. Nevoid basal cell carcinoma syndrome is estimated at 1 per 40,000 to 60,000 individuals with no sexual predilection. ${ }^{1,2}$ Pathogenesis of NBCCS occurs through molecular alterations in the dormant hedgehog signaling pathway, causing constitutive signaling activity and a loss of function in the tumor suppressor patched 1 gene, PTCH1. As a result, the inhibition of smoothened oncogenes is released, Gli proteins are activated, and the hedgehog signaling pathway is no longer quiescent. ${ }^{2}$ Additional loss of function in the suppressor of fused homolog protein, a negative regulator of the hedgehog pathway, allows for further tumor proliferation. The crucial role these genes play in the hedgehog signaling pathway and their mutation association with NBCCS allows for molecular confirmation in the diagnosis of NBCCS. Allelic losses at the PTCH1 gene site are thought to occur in approximately $70 \%$ of NBCCS patients. ${ }^{2}$

Diagnosis of NBCCS is based on genetic testing to examine pathogenic gene variants, notably in the PTCH1 gene, and identification of characteristic clinical findings. ${ }^{2}$ Diagnosis of NBCCS requires either 2 minor suggestive criteria and 1 major suggestive criterion, 2 major suggestive criteria and 1 minor suggestive criterion, or 1 major suggestive criterion with molecular confirmation. The presence of basal cell carcinomas (BCCs) before 20 years of age or an excessive numbers of BCCs, keratocystic odontogenic tumors (KOTs), palmar or plantar pitting, and first-degree relatives with NBCCS are classified as major suggestive criteria. ${ }^{2}$ Nevoid basal cell carcinoma syndrome patients typically have BCCs that crust, ulcerate, or bleed. Minor suggestive criteria for NBCCS are rib abnormalities, skeletal malformations, macrocephaly, cleft lip or palate, and desmoplastic medulloblastoma. ${ }^{2-4}$ Suppressor of fused homolog protein mutations may increase the risk for desmoplastic medulloblastoma in NBCCS patients. Our patient had 4 of the major suggestive criteria, including a history of KOTs, multiple BCCs, first-degree relatives with NBCCS, and palmar or plantar pitting (bottom quiz image), while having 1 minor suggestive criterion of frontal bossing.

Patients with NBCCS have high phenotypic variability, as their skin carcinomas do not have the classic features of pearly surfaces or corkscrew telangiectasia that typically are associated with BCCs. ${ }^{1}$ Basal cell carcinomas in NBCCS-affected individuals usually are indistinguishable from sporadic lesions that arise in sun-exposed areas, making NBCCS difficult to diagnose. These sporadic lesions often are misdiagnosed as psoriatic or eczematous lesions, and additional subsequent examination is required. The findings of multiple papules and plaques spanning the body as well as lesions with rolled borders and ulcerated bases, indicative of BCCs, aid dermatologists in distinguishing benign lesions from those of NBCCS. ${ }^{1}$

Additional differential diagnoses are required to distinguish NBCCS from other similar inherited skin disorders that are characterized by BCCs. The presence of multiple incidental BCCs early in life remains a histopathologic clue for NBCCS diagnosis, as opposed to Rombo syndrome, in which BCCs often develop in

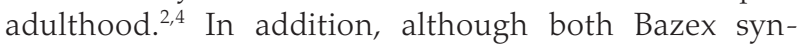
drome and Muir-Torre syndrome are characterized by the early onset of BCCs, the lack of skeletal abnormalities and palmar and plantar pitting distinguish these entities from NBCCS. ${ }^{2,4}$ Furthermore, though psoriasis also can present on the scalp, clinical presentation often includes well-demarcated and symmetric plaques that are erythematous and silvery, all of which were not present in our patient and typically are not seen in NBCCS. ${ }^{5}$

The recommended treatment of NBCCS is vismodegib, a specific oncogene inhibitor. This medication suppresses the hedgehog signaling pathway by inhibiting smoothened oncogenes and downstream target molecules, thereby decreasing tumor proliferation. ${ }^{6}$ In doing so, vismodegib inhibits the development of new BCCs while reducing the burden of present ones. Additionally, vismodegib appears to effectively treat KOTs. If successful, this medication may be able to suppress KOTs in patients with NBCCS and thus facilitate surgery. ${ }^{6}$ Additional hedgehog inhibitors include patidegib, sonidegib, and itraconazole. Patidegib gel $2 \%$ currently is in phase 3 clinical trials for evaluation of efficacy and safety in treatment of NBCCS. Sonidegib is approved for the treatment of locally advanced BCCs in the United States and the European Union and for both locally advanced BCCs and metastatic BCCs in Switzerland and Australia. ${ }^{7}$ Further research is needed before recommending antifungal itraconazole for NBCCS clinical use. ${ }^{8}$ Other medications for localized areas include topical application of 5 -fluorouracil and imiquimod. ${ }^{2}$

\section{REFERENCES}

1. Sangehra R, Grewal P. Gorlin syndrome presentation and the importance of differential diagnosis of skin cancer: a case report. J Pharm Pharm Sci. 2018;21:222-224

2. Bresler S, Padwa B, Granter S. Nevoid basal cell carcinoma syndrome (Gorlin syndrome). Head Neck Pathol. 2016;10:119-124.

3. Fujii K, Miyashita T. Gorlin syndrome (nevoid basal cell carcinoma syndrome): update and literature review. Pediatr Int. 2014;56:667-674.

4. Evans G, Farndon P. Nevoid basal cell carcinoma syndrome. GeneReviews [Internet]. University of Washington; 1993-2020. 
5. Kim WB, Jerome D, Yeung J. Diagnosis and management of psoriasis. Can Fam Physician. 2017;63:278-285.

6. Booms P, Harth M, Sader R, et al.Vismodegib hedgehog-signaling inhibition and treatment of basal cell carcinomas as well as keratocystic odontogenic tumors in Gorlin syndrome. Ann Maxillofac Surg. 2015;5:14-19.
7. Gutzmer R, Soloon J. Hedgehog pathway inhibition for the treatment of basal cell carcinoma. Target Oncol. 2019;14:253-267.

8. Leavitt E, Lask G, Martin S. Sonic hedgehog pathway inhibition in the treatment of advanced basal cell carcinoma. Curr Treat Options Oncol. 2019;20:84. 